\title{
Die Kompetenzverteilung in der österreichischen Verfassung von 1934
}

\author{
The Distribution of Competences in the Austrian Constitution 1934
}

Due to the authoritarian principle dominantly anchored in the constitution of 1934, federalism, which had still been prevalent in the previous constitution, was reduced - despite the designation 'federal state' for the new state system. Accordingly, a continuation of the tendency towards a centralisation of competences, which had already been evident in 1933, can be seen. Therefore, the competences of the federal government were strengthened, whereas the Länder were deprived of autonomy. At the same time, the system of competences was simplified and made more flexible. In reality, however, these provisions were overlaid by those of the Constitutional Transition Act, which made an unveiled chancellor dictatorship possible.

Keywords: authoritarian principle - centralisation - competences - constitution

\section{Vorbemerkung}

Bereits in der Phase des „Staatsstreiches auf Raten“ (Huemer), als die Regierung Dollfuß nach der sog. "Selbstausschaltung“ des Nationalrates am 4. März 1933 mit zahlreichen Verfassungsbrüchen die parlamentarische Demokratie durch eine Diktatur ersetzte, zeigte sich eine klare Tendenz zur Entmachtung der Länder, die freilich in politischer Hinsicht primär gegen das sozialdemokratische Wien gerichtet war - wurden doch die Materien des Sicherheitswesens und Staatsbürgerschaftsrechts bereits vor der Erlassung der neuen Verfassung einer Zentralisierung unterworfen. ${ }^{1}$

Diese Tendenz setzte sich auch in der Verfassung 1934 fort, wiewohl diese, so Adolf Merkl, dem föderalistischen Prinzip durchaus Raum gab, jedoch "nur in einem solchen Maße, daß das obers-

\footnotetext{
${ }^{1}$ Siehe dazu ausf. ReITER-ZATLOUKAL, Rechtsstellung. Auf diesen Beitrag stützen sich z.T. auch die folgenden Ausführungen.
}

te Leitmotiv des Verfassungswerks, nämlich die autoritäre Staatsführung, in keiner Weise in Frage gestellt“ wurde, hebe doch ein "nach den geschichtlichen Vorbildern durchgeführte[r] Typus des Bundesstaates" das "Autoritätsprinzip in dem modernen Sinne des Wortes" auf. ${ }^{2}$

Im Zuge der von den Parteien der Regierungskoalition schon seit den 1920er-Jahren ${ }^{3}$ geforderten Neugestaltung der österreichischen Verfassung musste auch die Kompetenzverteilung zwischen Bund und Ländern berufsständischautoritären Vorstellungen entsprechend umgestaltet werden. Diese Frage wurde im Ministerrat auch durchaus ausgiebig und kontroversiell diskutiert, die neuen Kompetenzbestimmungen fanden schließlich ihren Niederschlag in einem eigenen Hauptstück der Verfassung 1934 (3. Hauptstück) - wie zu zeigen ist.

In der Verfassungsrealität war die Neuordnung der Kompetenzen in der Verfassung 1934 aller-

\footnotetext{
${ }^{2}$ MerKL, Verfassung 202.

${ }^{3}$ Vgl. Reiter-ZatLOUKAL, Parlamentarismus.
} 
dings durch das Regelwerk der „Übergangsverfassung ", 4 also des Verfassungs-Überleitungsgesetzes (V-ÜG) 1934,5 überlagert und kam nur teilweise zum Tragen.

\section{Verfassungsdiskussion 1934}

Im Jänner 1934 legte der mit der Verfassungsreform betraute Minister Otto Ender einen Entwurf für die neue Verfassung vor, ${ }^{6}$ der trotz der bisherigen Zentralisierungsmaßnahmen der Regierung grundsätzlich am bundesstaatlichen Charakter Österreichs festhielt, weil dies, so Ender im Ministerrat, ${ }^{7}$, die geschichtliche Entwicklung Österreichs verlangt". Daher wurde auch an der Teilung der Gesetzgebung- und Vollziehungsgewalt zwischen Bund und Ländern „ungefähr in der bisherigen Art" festgehalten. Eine Änderung gäbe es jedoch im Bereich des Schulwesens, da bei der Schaffung des B-VG die „Kompetenzfrage auf dem Gebiet des Schulwesens nicht entschieden" worden sei - was dazu geführt habe, dass „gewisse Materien nur durch sogenannte paktierte Gesetze geregelt werden konnten“, ein ",allgemein als sehr unerwünscht bezeichneter Zustand“. Hier schaffe der Entwurf „Klarheit“, indem er „die Hochschulen, die mittleren Lehranstalten, gewisse Fachhochschulen usw. ausschließlich dem Bunde zu[weise]“. Die sonstigen Angelegenheiten des Schul-, Erziehungs- und Volksbildungswesens einschließlich der Schulaufsicht würden nun der Grundsatzgesetzgebung des Bundes zufallen. „Wenn unsere Gesetzgebung“, so Ender, „die Kunst wirklicher Grundsatzgesetzgebung wieder erlernt, eine Kunst, die ihr vor 50 bis 70 Jahren zu eigen war, so kann diese Lösung sehr glücklich sein“, denn der Bund könne „seine berechtigten Interessen

\footnotetext{
${ }^{4}$ WIEDERIN, Bundesstaatlichkeit 32.

${ }^{5}$ Art. III § 56 Abs. 3 V-ÜG, BGBl. II 75/1934.

${ }^{6}$ Ausf. EtLINGER, Landesverfassungen 32-35.

${ }^{7}$ MRP Nr. 919, 1. 2. 1934, Beil. A, Vortrag für den Ministerrat, NECK, WANDRUSZKA, Protokolle VIII/5 510f.
}

voll wahren und die Länder kommen in der Ausführungsgesetzgebung zu ihrem Recht“.

In weiterer Folge wurde dieser Verfassungsentwurf im Ministerrat beraten, ${ }^{8}$ wobei sich die Zentralisten (v.a. die Minister Fey, Kerber, Neustädter-Stürmer) und die Vertreter eines modifizierten Föderalismus (die Minister Schuschnigg, Schmitz und Buresch) gegenüberstanden. Die Diskussionen in diesem Zusammenhang drehten sich neben den Kompetenzbestimmungen auch u.a. um den Modus der Bestellung des Landeshauptmannes und die Organisation der Bezirkshauptmannschaften, worauf hier nicht näher eingegangen werden kann. ${ }^{9}$ Was die Kompetenzverteilung betrifft, sprach sich Odo Neustädter-Stürmer gegen die im Entwurf vorgesehene taxative Aufzählung der Bundeskompetenzen und damit verbunden die „allgemeine Kompetenz" der Länder aus und forderte eine Generalklausel zugunsten des Bundes. Für Justizminister Kurt Schuschnigg war es ,selbstverständlich, dass heute die Kompetenzen des Bundes sehr ausgedehnt sein" müssten, man könne es aber „im allgemeinen bei der bisherigen Ordnung belassen“. Er betonte allerdings, dass das „heutige System der Gegenüberstellung von Grundsatzgesetzgebung des Bundes und Ausführungsgesetzgebung der Länder [...] zu manchen Unzukömmlichkeiten geführt habe“. Jedenfalls könne man die Kompetenzen des Bundes weiterhin taxativ aufzählen und „brauche keine Sorge zu haben, dass [diese] die Macht der Länder überwuchern werde".

Für Richard Schmitz, damals Kanzleramtsminister, war es überhaupt „,schwer zu sagen, wo der Bundesstaat aufhöre und der Einheitsstaat anfange", die Zuteilung von Gesetzgebungskompetenzen an die Länder sei aber erforderlich, wenn man den bundesstaatlichen Charakter aufrechterhalten wolle. Dem „Subsistarprinzip“ ent-

\footnotetext{
${ }^{8}$ Für alle Zitate siehe MRP Nr. 920, 20. 3.-29. 3. 1934, NECK, WANDRUSZKA, Protokolle VIII/6 152-157.

${ }^{9}$ Ausf. EtLinger, Landesverfassungen 35-42.
} 
sprechend habe jede "Gemeinschaft [...] jene Aufgaben zu erfüllen, zu deren Besorgung sie mit eigenen Kräften fähig sei“. Die Kompetenzverteilung müsse von diesem Gesichtspunkt beurteilt und so gestaltet werden, "dass die Landeskompetenzen wirklich in den inneren Wirkungskreis der Länder hineinpaßten“. Dem Bund sollte jedenfalls die Möglichkeit zukommen, „bei Mängeln der Landesgesetzgebung [...] korrigierend einzugreifen“, denn "die wirkliche Schwierigkeit" liege nicht in der Kompetenzverteilung, sondern im „Mangel eines Sanktionsrechtes gegenüber Landesgesetzen“. Daher müsse ein solches Sanktionsrecht des Bundes „die Wahrung der vom Bund zu beobachtenden Belange sicherstellen“. Auch Kanzler Engelbert Dollfuß sprach sich für eine Sanktion der Landesgesetze aus und verwies auf die diesbezüglichen Normen in der Monarchie. Ender selbst erklärte sich mit der „Wiedereinführung des Sanktionsrechtes“ einverstanden, wenn man „den Ländern die früher besessene Gesetzgebungskompetenz wieder zuerkenne“ und „die Erteilung der Sanktion an eine bestimmte Frist binde“.

Hinsichtlich der Vollziehungskompetenzen warf Neustädter-Stürmer in der Diskussion die Frage auf, ob es in der neuen Verfassung „überhaupt noch notwendig sei, eine Grenze zwischen der Vollziehung des Bundes und jener des Landes zu ziehen“, weil nach dem vorliegenden Entwurf, „von geringen Ausnahmen abgesehen dem Land ohnedies in jedem Fall die Vollziehung zukomme“. Bei der Unterscheidung in Bundes- und Landesvollziehung handle sich um „ein Überbleibsel aus der Zeit der verländerten Verwaltung, in der zwischen unmittelbarer und mittelbarer Bundesverwaltung unterschieden" worden sei, woran aber nicht festgehalten werden solle. Vielmehr würde genügen, „lediglich zwischen der Gesetzgebung des Bundes und der Länder zu unterscheiden“.

Sicherheitsminister Emil Fey schlug vor, „in jenen Angelegenheiten der Bundesgesetzgebung, in denen es möglich und zweckmäßig ist, auch die Erlassung der Durchführungsbestimmungen dem Bund vorzubehalten, dem es dann freistehen solle, den Vollzug durch eigene Organe zu besorgen." Fey lehnte es überhaupt ab, "gewisse Bundesagenden, insbesondere betreffend die öffentliche Sicherheit, durch Landesorgane besorgen zu lassen, wenn dem Bund nicht gleichzeitig ein Einfluss auf diese Organe zustünde", wie dies vorbildlich bei den 1933 geschaffenen Sicherheitsdirektoren, die direkt dem Bundeskanzleramt unterstellt waren, der Fall sei. Man müsse dem Bund daher auch einen Einfluss auf die Enthebung der nach dem Entwurf für Sicherheitsangelegenheiten zuständigen Bezirkshauptmänner einräumen und diese entweder zu Bundesbeamten (oder zu reinen Landesbeamten bei gleichzeitigem Entzug dieser Agenden) machen. $\mathrm{Zu}$ dieser „Lösung müsse man kommen, wenn man ungesunde Verhältnisse vermeiden“ wolle.

Für Innenminister Robert Kerber wiederum hatte sich das Institut der Grundsatzgesetzgebung „nicht bewährt“, und überdies sei das „Nebeneinander" von Bundes- und Landesgesetzgesetzgebung einerseits sowie der Grundsatzgesetzgebung des Bundes andererseits „überaus kompliziert und kostspielig“. Er schlug daher vor, nur "einige wenige Landeskompetenzen“ vorzusehen und „alles übrige als Kompetenz des Bundes zu erklären“, denn die „notwenige Freiheit in den Ländern" sei gewahrt, wenn die Durchführung durch Verordnungen der Landesregierung erfolge. Staatssekretär Franz Glas trat ebenfalls dafür ein, „das öffentliche Leben so billig als möglich zu gestalten", plädierte aus diesem Grund aber dafür, sich an die „bereits bestehende Organisation" zu halten.

Finanzminister Karl Buresch betonte, dass den Ländern jedenfalls eine „gewisse Selbständigkeit" zukommen müsse, wenn denn Österreich ein Bundesstaat sein solle. Es sei aber erforderlich, die Finanzverfassung „einer genauen Prüfung“ zu unterziehen, denn zwecks Gesundung der Wirtschaft müsse man dem Bund auch hier einen „möglichst weite[n] Wirkungskreis“ ein- 
räumen. Hinsichtlich der Grundsatzgesetzgebung des Bundes führte er die Einschätzung, dass sie „die in sie gesetzten Erwartungen“ nicht erfüllt hätte, auf die ,früheren Parteiverhältnisse" zurück. Wenn aber die Zentralgewalt wieder „fester gefügt" sein werde, dann ließe sich auch auf dem Gebiet der Grundsatzgesetzgebung „ein guter Weg“ finden, der zur Schaffung „eines einheitlichen Bundesrechts" führen könne. Ein wesentliches Anliegen bei der Kompetenzverteilung sei aber, „daß beim Bund jene Angelegenheiten zu verbleiben hätten, die vom Bund alleine geführt werden sollten", wie etwa das Elektrizitätswesen.

Ender verwehrte sich gegen eine „Unterschätzung des Werts der Grundsatzgesetzgebung" und gegen die „Umkehrung des Systems“ der taxativen Aufzählung der Bundeskompetenzen mit einer Generalklausel zugunsten der Länder. Außerdem müsste ein solcher Vorschlag mit den Ländern besprochen werden, andernfalls dies zu „schweren Auseinandersetzungen mit den Ländern führen" müsse. Solche Verhandlungen machte Ender sogar zur „Voraussetzung für seine weitere Mitarbeit". In der Frage der Vollziehung der Länder vertrat er als Verfechter des Föderalismus die Ansicht, dass „dort, wo das Land die Vollziehung habe, ihm auch das Recht zukomme, Durchführungsverordnungen zu erlassen“. Ender sah daher auch kein Problem darin, das gesamte Elektrizitätswesen dem Bund zu überweisen, wenn die Vollziehung bei den Ländern verbleibe, ebenso auch die Straßenpolizei, wiewohl er bezweifelte, dass der Bund in der Lage sei, „die mit dieser Materie zusammenhängenden Fragen ganz befriedigend zu lösen“. Darüber hinaus habe man nun das gesamte Dienstrecht der öffentlichen Angestellten dem Bund überantwortet, womit der ,"ungesunde Zustand“ beseitigt werden könne, dass es in den Ländern neben Landesangestellten auch Bundesangestellte gebe; die Vollziehung „,werde man dem Land jedoch nicht wegnehmen können".
Wiewohl die Verhandlungen im Ministerrat bereits klar die Weichen in Richtung eines Föderalismusabbaus und der weiteren Zentralisierung gestellt hatten, ohne dass dies mit Landesrepräsentanten akkordiert worden wäre, stellte Bundeskanzler Dollfuß schließlich in Aussicht, die Kompetenzfrage mit den Landeshauptmännern zu besprechen. Wurde am 28. März 1934 noch in den Medien betont, dass „die Frage des Einbaues der gesetzgebenden Kompetenzen der Länder in die künftige Verfassung“ noch „,[n]icht restlos geklärt" sei,10 so hieß es bereits einen Tag später „Mit den Ländern einig“."11 Berichtet werden konnte nun, dass die Ländervertreter die neue Verfassung nach einer mehrstündigen Besprechung mit Dollfuß und Ender gebilligt hätten, womit die Verfassung „nicht nur von dem Willen der Bundesregierung, sondern auch der Ländervertretungen getragen" wäre. ${ }^{12}$

\section{Maiverfassung 1934}

Die von Regierung bereits Ende April mittels einer Verordnung nach dem Kriegswirtschaftlichen Ermächtigungsgesetz von 1917 oktroyierte neue Verfassung für den „christlichen, deutschen Bundesstaat auf ständischer Grundlage“ (Präambel) wurde am 1. Mai 1934 feierlich proklamiert. Obwohl die Bezeichnung „Bundesstaat Österreich" anders vermuten lässt und die Bundesstaatlichkeit durchaus ein „wichtiges Gliederungsprinzip" geblieben war, so erschien sie "der Sache nach aber erheblich abgeschwächt", 13 vertrug sich doch ein wirklicher Föderalismus, wie bereits angesprochen, nicht mit der autoritär-zentralistischen Staatskonzeption, weshalb

\footnotetext{
${ }^{10}$ Die Stunde, 28. 3. 1934, 1 (Einzelheiten der Verfassung).

${ }^{11}$ Salzburger Chronik für Stadt und Land, 29. 3. 1934, 1 (Mit den Ländern einig).

${ }^{12}$ Neues Wiener Journal, 29. 3. 1934, 2 (Länder- und Wirtschaftsvertreter billigen neue Verfassung!).

${ }^{13}$ WIEDERIN, Bundesstaatlichkeit 34.
} 
die Länder Kompetenzen einbüßten. Auch wurde die Mitsprache der Länder im Bundesgesetzgebungsverfahren erheblich reduziert, und sie verloren ihre Autonomie in der Landesgesetzgebung und -organisation, wie gezeigt wird.

\subsection{Kompetenzverteilung}

Wie Merkl feststellte, habe die gesetzgeberische Aufgabe bei der Neuregelung der Kompetenzen „in einer großzügigen Kommassation der Kompetenzen von Bund und Ländern, wennmöglich in einer Verminderung der Zahl der Kompetenztypen, die durch die Varianten der Hauptwege der Art. 10-15 [B-VG] auf ein volles Dutzend angeschwollen waren, ${ }^{14}$ und jedenfalls in einer Zusammenfassung mancher bisheriger übermäßig spezialisierter und daher schwer abgrenzbarer Kompetenzfälle zu größeren Agendenkomplexen" bestanden. ${ }^{15}$ Dies war auch im Wesentlichen gelungen, denn die in der Verfassung festgelegte Kompetenzverteilung weist im Vergleich zu der ihrer Vorgängerin eine Vereinfachung und eine Verminderung der Zahl der Kompetenztypen auf. Merk ${ }^{16}$ zufolge sei auch „rückhaltslos anzuerkennen, daß das neue System der Kompetenzverteilung zwischen Bund und Ländern viele Unebenheiten der durch die beiden Verfassungsnovellen [...] 1925 und [...] 192917 überflüssig und übermäßig kompliziert gewordenen Kompetenzordnung ausgeglichen und manche Bizarrerien dieser Kompetenzordnung, die ihresgleichen auf der Erde gesucht [hätten], beseitigt" habe. Für Merkl war dieses „irrationale Flickwerk der bisherigen Kompetenzbestimmungen größtenteils [ein] typisches Parteienkompromiß“, über das nun "ein autoritäres Regime einfach hinweggehen" habe können.

\footnotetext{
${ }^{14}$ Siehe WERNDL, Kompetenzverteilung.

${ }^{15}$ MERKL, Verfassungsrecht 268; auch als DERS., Verfassung 57.

${ }^{16}$ Ebd. 57f.

${ }^{17}$ Siehe dazu die Beiträge von Stephan HinghoferSzalkay und Gerald Kohl in diesem Band.
}

Nun habe die „annähernde Gleichschaltung des politischen Systems in Bund und Ländern [...] auch einer diffizilen Kompetenzabgrenzung den ursprünglichen Sinn genommen“. Es würde nämlich „bei der politischen Gleichrichtung der Bundes- und Ländergewalten im sachlichen Ergebnis kaum etwas ändern", ob nun der Bund oder ein Land über diese oder jene Kompetenz verfüge. Für Merkl war es allerdings „nicht ersichtlich“, ob für die „neue, aber in den Grundlagen doch unveränderte Kompetenzordnung“ nur „das Beharrungsvermögen der letzten noch jungen und mühsam ausgeklügelten Kompetenzordnung ausschlaggebend" war, oder ob die „Ressortinteressen der Bundes- und Landesbürokratien einfach an die Stelle der gegensätzlichen Parteiinteressen getreten " seien und sich deren "kompliziertes, in der Bundesverfassung 1920 verankertes Kompromiß zu eigen gemacht" hätten.

Im Einzelnen weist die Verteilung der Zuständigkeiten in der Tat "manche Veränderung" auf. ${ }^{18}$ So wurden zwar die Bundeskompetenzen (Art. 34) weiterhin in einem „Riesenkatalog ${ }^{\prime 19}$ taxativ aufgezählt, dies aber zu Lasten der Länder, denn die in Form einer Generalklausel umschriebenen Landeskompetenzen (Art. 40) wurden dadurch nun erheblich reduziert. Die dem Bund zugewiesenen Kompetenzen waren jedenfalls ihrer ,juristischen, kulturellen, wirtschaftlichen und politischen Bedeutung nach [...] die wichtigsten“, ${ }^{20}$ darunter etwa: „Aufbau, Einrichtung und Aufgaben der ständischen Verwaltung", Bundesfinanzen, Sicherheitswesen, Gewerbe, Handel und Industrie, Verkehrswesen usw.

Die schon vor Erlassung der Verfassung feststellbare "Tendenz zur Verbundlichung “21 von Angelegenheiten, an denen bisher die Länder teilgehabt hatten, zeigt sich etwa - in Fortset-

\footnotetext{
18 JÄCKL, Verfassung 105.

${ }^{19}$ KULISCH, Studien 169.

${ }^{20}$ Ebd.

${ }^{21}$ MERKL, Verfassung 59.
} 
zung der sich schon 1933 manifestierenden Tendenz - klar nicht nur im Bereich des Staatsbürgerschaftswesens, ${ }^{22}$ sondern auch des Elektrizitätswesens sowie in Teilen des Krankenanstaltswesens und der Landeskultur. Überdies wurden nun einige Angelegenheiten aufgenommen, die „bisher nicht in der Verfassung aufgezählt" worden waren, ${ }^{23}$ so etwa das Rundfunkwesen, die Fremdenverkehrsförderung (soweit sie sich auf das ganze Bundesgebiet bezog), das Messewesen, die Unterbringung ausgedienter Soldaten im öffentlichen Dienst, das Buchmacher- und Totalisateurwesen.

Diese „starre Zentralisation" wurde allerdings dadurch durchlöchert, dass durch einfache Bundesgesetze die Landesgesetzgebung ermächtigt werden konnte, ,zu näher zu bezeichnenden Bestimmungen Ausführungsbestimmungen zu erlassen", womit sich, wie Kulisch kritisierte, ${ }^{24}$ die eigentliche Bundesgesetzgebung zu einer „bloßen Grundsatzgesetzgebung des Bundes verwandelt" habe. Daher biete allein die „Einsicht der Bundesregierung [...] eine politische Gewähr dafür, daß sie die Gesetzesinitiative nicht leichten Herzens ergreifen werde", damit nicht „die Einheitlichkeit der Rechtssatzung in den im Art. 34 aufgezählten Gegenständen [... gefährdet]" werde.

Im Bereich des Schulwesens (Art. 37) wurden nun die Kompetenzen des Bundes durch Wegfall der bisherigen ,paktierten Gesetzgebung “25

\footnotetext{
${ }^{22}$ Nun waren die Staatsbürgerschaftsangelegenheiten nämlich in Gesetzgebung und Vollziehung Bundessache, sodass der Bund auch über Fragen der Landesbürgerschaft zu entscheiden hatte. Diese Bestimmungen sollten jedoch erst mit dem Inkrafttreten eines neuen, den Grundsätzen der Verfassung 1934 entsprechenden Bundesgesetzes wirksam werden, das bis zum "Anschluss" nicht mehr erging; siehe dazu ausf. REITER-ZATLOUKAL, Staatsbürgerschaftsrecht.

${ }^{23}$ Kleine Volkszeitung, 1. 5. 1934, Nr. 118, 9.

${ }^{24}$ KULISCH, Studien 169.

${ }^{25}$ Also: übereinstimmende Gesetze des Bundes und der Länder.
}

gestärkt: Dem Bund kam insbes. in Sachen der Hoch- und Mittelschulen die Gesetzgebung zu, während für Hauptschulen, Volksschulen und gewerbliche sowie landwirtschaftliche Fortbildungsschulen dem Bund bloß die Grundsatzgesetzgebung, den Ländern jedoch die Ausführungsgesetzgebung oblag.

Übernommen aus der Rechtslage der 2. B-VGNovelle wurde allerdings die „Bedarfsgesetzgebung“ des Bundes, der zufolge das Verwaltungsverfahren, die allgemeinen Bestimmungen des Verwaltungsstrafrechtes, das Verwaltungsstrafverfahren und die Verwaltungsvollstreckung durch Bundesgesetz geregelt werden konnten, „soweit ein Bedürfnis nach Erlassung einheitlicher Vorschriften" (Art. 35) bestand.

Ebenfalls im Einklang mit dem B-VG stand der kompetenzrechtliche Grundtypus der Gesetzgebung und Vollziehung des Landes in Form der bereits angesprochenen Generalklausel (Art. 40 Abs. 6), womit die Kompetenz des Bundes „positiv, die der Länder negativ festgestellt" erscheint. ${ }^{26}$ Der Wirkungskreis des Bundes war freilich so groß, „das für die Länder nichts von Belang übrig“ blieb. Dazu kam, dass der Bund „durch Änderung seiner eigenen Verfassung seine Zuständigkeit auf Kosten der Länder jederzeit erweitern" konnte. Daher könne, so Kulisch, dem Art. 40 Abs. 6 ,keine allzu große politische Bedeutung zuerkannt werden, aber seine sachliche Brauchbarkeit muss voll und ganz anerkannt werden“. ${ }^{27}$

Am meisten allerdings "springt in die Augen“, so Hugo Jäckel über die Kompetenzneuregelung, ${ }^{28}$ dass es "Angelegenheiten, in denen die Gesetzgebung Sache des Bundes, die Vollziehung Sache der Länder ist, in Zukunft nicht mehr geben wird“. Es entfiel nämlich die „ent-

\footnotetext{
${ }^{26}$ KULISCH, Studien 173.

${ }^{27}$ Ebd. 174.

28 JÄCKL, Verfassung 106.
} 
behrlich gewordene ${ }^{\iota_{29} 9}$ geteilte bzw. gespaltene Kompetenz zwischen Bund und Ländern in der bisherigen Form (Gesetzgebung Bundessache, Vollziehung Landessache, Art. 11 B-VG).

Beibehalten wurde jedoch die Verteilung, dass dem Bund die Grundsatzgesetzgebung und dem Land die Erlassung von Ausführungsgesetzen sowie die Vollziehung (Art. 36) oblag, wobei nun etwa auch das bisher in die ausschließliche Kompetenz des Bundes fallende Heimatrecht diesem nur mehr für die Grundsatzgesetzgebung zugewiesen wurde, was in diesem Punkt freilich eine Verstärkung der Landeskompetenz bedeutete. Die Vollziehung der Ausführungsgesetze zu den Grundsatzgesetzen oblag der Landesregierung und den in ihrem Namen handelnden Bezirkshauptmannschaften. Es galt also, wie es Wiederin auf den Punkt bringt, der Grundsatz, dass „die Gesetze vollzieht, wer sie macht" ${ }^{\prime 3}$

Wie Ender dazu in seiner Erläuterung der Verfassung ausführte, sollte mit dieser Bestimmung der Bundesgesetzgeber zur „wirklichen Grundsatzgesetzgebung zurückzukehren“, wozu er sich nun „leicht entschließen“ könne, da ihm ja ein "Sanktionsrecht" in Form einer Zustimmung des Bundeskanzlers zu Landesgesetzen (siehe wieter unten) zustehe, das ihm schließlich „nicht zuletzt aus diesem Grunde eingeräumt " worden sei. ${ }^{31}$ Nach Art. 39 hatte sich der Bund daher bei der Grundsatzgesetzgebung tatsächlich nur ,auf die Festsetzung der allgemeinen Grundzüge der betreffenden Angelegenheiten zu beschränken“, womit „der bisherigen Übung entgegen gesteuert werden [sollte], durch Detailregelung der Grundsatzgesetzgebung des Bundes das Blankett für die Ausführungsgesetzgebung der Länder soweit auszufüllen, daß die Länder in ihren

\footnotetext{
${ }^{29}$ MerKL, Verfassung 59. Die bisher in die autonome Landesverwaltung fallenden Angelegenheiten würden nun von den Ländern in Form der mittelbaren Bundesverwaltung besorgt.

${ }^{30}$ WIEDERIN, Bundesstaatlichkeit 34.

${ }^{31}$ ENDER, Verfassung 8.
}

Ausführungsgesetzen auf die Normierung von Nebensächlichkeiten beschränkt bleiben“32 was für Kulisch eine wichtige Neuerung darstellte, um den „verfassungspolitischen Zweck dieser Kompetenztype " zu erreichen. ${ }^{33}$

Völlig neu war die Möglichkeit, dass sich der Bund und die Länder ihre verfassungsmäßigen Kompetenzen gegenseitig delegieren konnten, ohne dass dazu eine Verfassungsänderung erforderlich war, wodurch die Kompetenzbestimmungen der Verfassung nun "biegsamer" wurden. ${ }^{34}$ Die Übertragung der Gesetzgebungsbefugnisse an den Bund mit Wirkung für alle Länder erfolgte, wenn der Länderrat (siehe dazu weiter unten) einen derartigen Beschluss mit Zweidrittelmehrheit fasste (Art. 41 Abs. 2). Dies stellte im Übrigen auch den einzigen Fall dar, dass ein „Vorberatendes Organ“ aus eigener Initiative ein Gesetz im materiell-rechtlichen Sinn beschließen konnte. ${ }^{35}$ Für Merk1 ${ }^{36}$ lagen die „,besonderen Motive dieser eigentümlichen gegenseitigen Delegationsbefugnis" zwar nicht klar zutage, aber die Tendenz sei „offensichtlich, die Kompetenzlage möglichst leicht späterhin zutage tretenden Augenblicksbedürfnissen anzupassen".

"Alle diese neuen Bestimmungen“, würden freilich, so Jäckel, ${ }^{37}$ ",an Bedeutung bei weitem durch die [bereits erwähnte] Bestimmung übertroffen", dass alle Gesetzesbeschlüsse der Landtage nur kundgemacht werden durften, wenn der Bundeskanzler zugestimmt hatte (Art. 111 Abs. 2), denn damit trat ", an die Stelle des bisherigen suspensiven Vetos der Bundesregierung das absolute Veto des Bundeskanzlers", was „Zweifellos einen stark zentralistischen Einschlag" der Verfassung bedeute.

\footnotetext{
${ }^{32}$ MerKL, Verfassung 60.

${ }^{33}$ KULISCH, Studien 170.

${ }^{34} \mathrm{Ebd}$.

35 POLASCHEK, Föderalismus 154.

${ }^{36}$ MERKL, Verfassung 61.

37 JÄCKL, Verfassung 107.
} 
Wie Merkl ${ }^{38}$ hinsichtlich der ,starken Zentralisation" kritisch feststellte, hätte diese freilich, „wenn überhaupt ein föderalistischer Einschlag der Verfassung erhalten werden sollte, von der Herausgabe mancher bisher dem Bund vorbehalten gewesener Kompetenzstücke an die Länder begleitet werden können, da ja in der ungewöhnlichen Zentralisation des Organapparates, in der straffen Subordination der Ländergewalten unter die Bundesgewalt, genügende Garantien gelegen hätten, daß die Landeskompetenzen im Sinne der zentralen Staatsgewalt ausgeübt werden". Auch beanstandete Merkl, dass die „so günstige Gelegenheit zum Ausschluß des unfruchtbaren Rechtsstreits, des Kompetenzstreits, leider nur unvollkommen und vereinzelt benützt" und sogar „neue problematische Kompetenzgrenzen geschaffen" worden seien. Außerdem habe die Verfassung sich der "ganzen Anlage nach [...] auf eine Auseinandersetzung der Kompetenzen zwischen Bund und Ländern [beschränkt] und [...] der auf Grund dieser Kompetenzordnung erfolgenden Gesetzgesetzgebung die Kompetenzverteilung zwischen dem Staat [in dessen Erscheinungsformen Bund und Länder] und den Ständen [überlassen]".

\subsection{Bundesstaatlichkeit und Gesetzgebung}

\subsubsection{Bundesgesetzgebung}

Der Ablöse der parlamentarischen Demokratie und dem Rückbau des Föderalismus durch einen autoritär-zentralistischen Staat sowie dem ständestaatlichen Grundgedanken der Verfassung entsprach auch das neue Verfahren der ordentlichen Bundesgesetzgebung. Das alleinige Recht der Gesetzesinitiative stand der Bundesregierung $\mathrm{zu}$, von der Gesetzentwürfe an die vier „Vorberatenden Organe“ übermittelt wur-

\footnotetext{
${ }^{38}$ MERKL, Verfassung 57f., 61.
}

den, nämlich den Staatsrat, 39 Bundeskulturrat, ${ }^{40}$ Bundeswirtschaftsrat ${ }^{41}$ sowie Länderrat, in welchen jedes Land den Landeshauptmann und den Finanzreferenten entsendete. Diese Vorberatenden Organe erstatteten Pflicht- oder Freigutachten, an welche die Regierung aber nicht gebunden war. Nach Einlangen der Gutachten wurde der Gesetzentwurf dann von der Bundesregierung dem aus Mitgliedern der „Vorberatenden Organe“ zusammengesetzten Bundestag zur Beschlussfassung ohne Abänderungsmöglichkeit vorgelegt.

Dem Länderrat oblag die Begutachtung von Gesetzesentwürfen vom Standpunkt der Landesinteressen; verpflichtend war der Länderrat aber nur zur Begutachtung heranzuziehen, wenn die

\footnotetext{
${ }^{39}$ Der Staatsrat bestand aus 40 bis 50 vom Bundespräsidenten unter Gegenzeichnung des Bundeskanzlers zu ernennenden Mitgliedern und sollte dazu Stellung nehmen, ob der Entwurf den "Anforderungen der Staatshoheit und des Gemeinwohles wie auch jenen einer zweckmäßigen Gesetzesvollziehung“ entsprach. Die Mitglieder des Staatsrates wurden auf 10 Jahre berufen und hatten „verdiente, charaktervolle Bundesbürger zu sein, von denen nach ihrem bisherigen Verhalten und nach ihren bisherigen Leistungen volles Verständnis für die Bedürfnisse und die Aufgaben des Staates zu erwarten" war.

${ }^{40}$ Der Bundeskulturrat setzte sich aus 30 bis 40 Vertretern der gesetzlich anerkannten Kirchen und Religionsgesellschaften sowie des Schul-, Erziehungsund Volksbildungswesens, der Wissenschaft und der Kunst zusammen und hatte sein Gutachten vom Standpunkt der kulturellen Interessen abzugeben.

${ }^{41}$ Der Bundeswirtschaftsrat bestand aus 70 bis 80 von den „berufsständischen Hauptgruppen“ zu entsendenden Mitgliedern und sollte sein Gutachten vom Standpunkt der wirtschaftlichen Interessen abgeben. Die sieben zu errichtenden berufsständischen Hauptgruppen bzw. Berufsstände waren: Land- und Forstwirtschaft; Industrie und Bergbau; Gewerbe; Handel und Verkehr; Geld-, Kredit- und Versicherungswesen; freie Berufe; öffentlicher Dienst. Die Entsendung der Bundeswirtschaftsräte sollte wie die Berufung der Bundeskulturräte durch ein Bundesgesetz geregelt werden, das die Beschickung mit „vaterlandstreuen“ Mitgliedern (worunter Mitglieder der „Vaterländischen Front" verstanden wurden) gewährleistete.
} 
Bundesregierung vorhatte, einen Landtag aufzulösen. Abgesehen von der Begutachtung kam dem Länderrat darüber hinaus in zwei Fällen ein absolutes Veto in der Bundesgesetzgebung $\mathrm{zu}$, nämlich einerseits bei der Erlassung von Grundsatzgesetzen des Bundes, die für die Länder eine Frist für Ausführungsgesetze festlegten, die weniger als sechs Monate oder mehr als ein Jahr betrug. Erstattete der Länderrat kein zustimmendes Gutachten, dann trat die Fristbestimmung nicht in Kraft. Ein zustimmendes Gutachten war andererseits erforderlich, damit ein Gesetz über die Einrichtung und Geschäftsführung der Landeshauptmannschaft in Kraft treten konnte. Andererseits konnte der Länderrat, wie bereits erwähnt, mit Wirksamkeit für alle Länder deren Gesetzgebungskompetenz für eine bestimmte Angelegenheit dem Bund übertragen. Damit waren - im Unterschied zum BVG 1920 i.d.F. 1929 - die Mitwirkungsrechte der Länder an der Bundesgesetzgebung im Sinne der intendierten Zentralisierung also insgesamt deutlich eingeschränkt.

\subsubsection{Landesgesetzgebung}

Auch die Landesgesetzgebung erfuhr durch die Verfassung 1934 tiefgreifende Veränderungen, und zwar im Sinne des Einbaus der Berufsstände sowie der Zentralisierung und Verankerung des autoritären Prinzips, wodurch die Länder ihren bisherigen autonomen Status verloren. Die Landtage gingen entsprechend dem berufsständischen Prinzip der Verfassung nicht aus Wahlen hervor, sondern wurden nach der Verfassung analog zum Bundeskulturrat und Bundeswirtschaftsrat gebildet (Art. 108 Abs. 2). Folglich waren die Landtage „Delegiertenversammlungen der Berufsstände und der diesen entsprechenden kulturellen Gemeinschaften im Lande“ ${ }^{4}$

Der Gang der Landesgesetzgebung war dem der Bundesgesetzgebung insofern nachgebildet, als

\footnotetext{
${ }^{42}$ MERKL, Verfassung 94.
}

entsprechend dem autoritären Prinzip die Gesetzesinitiative nur der Landesregierung zustand, der Landtag war allerdings sowohl begutachtendes als auch beschließendes Organ. Wie der Bundestag auf Bundesebene konnte er aber ebenfalls nur die Vorlage der Landesregierung ohne Verhandlungen und ohne Abänderung annehmen oder ablehnen (Art. 109).

Einen massiven Eingriff in die Autonomie der Länder stellte freilich die bereits genannte Bestimmung dar, dass Landesgesetze der Zustimmung des Bundeskanzlers bedurften, denn ein Gesetzesbeschluss des Landtages durfte nur kundgemacht werden, wenn der Bundeskanzler zugestimmt hatte, allenfalls auch nur durch Verschweigung. Die Zustimmung galt nämlich als erteilt, wenn der Bundeskanzler nicht binnen sechs Wochen ab Einlangen des Gesetzesbeschlusses beim Bundeskanzleramt dem Landeshauptmann über die Verweigerung der $\mathrm{Zu}$ stimmung Mitteilung gemacht hatte. Schuschnigg begründete diese Maßnahme mit der notwendigen Bedachtnahme auf wichtige Gemeininteressen im ganzen Bundesgebiet. ${ }^{43}$ Merkl ${ }^{44}$ erblickte freilich in dieser „essentiellen Steigerung des Bundeseinflusses" schlichtweg eine Aufhebung der "Autonomie der Länder in der Sphäre der formellen Gesetzgebung", denn der Bundeskanzler war „Faktor der Landesgesetzgebung" geworden, womit die Landesgesetzgebung aufgehört habe, eine ausschließliche Angelegenheit der Länder zu sein.

Außerdem sah das V-ÜG $1934^{45}$ für die Landtage vor, dass infolge des noch nicht abgeschlossenen Aufbaus der Berufsstände eine provisorische Bestellung der Landtagsmitglieder durch den Landeshauptmann zu erfolgen hatte, wobei dieser sowohl die Gesamtzahl als auch die konkrete Aufteilung der Landtagsmitglieder auf die kulturellen Gemeinschaften und beruflichen Or-

\footnotetext{
${ }^{43}$ SCHUSCHNIGG, Verfassung 68.

${ }^{44}$ MERKL, Verfassung 96.

${ }^{45}$ Art. III § 56 Abs. 3 V-ÜG, BGBl. II 75/1934.
} 
ganisationen festlegte, wofür er einer Genehmigung des Bundeskanzlers bedurfte (§ $29 \mathrm{~V}-\mathrm{U} G$ ).

\subsection{Bundesstaatlichkeit und Verwaltung}

Zur Besorgung der Geschäfte der Bundesverwaltung waren nach der Verfassung 1934 wie auch zuvor das Bundeskanzleramt und die übrigen Bundesministerien sowie die ihnen unterstellten Ämter berufen.

Im Bereich der Landesverwaltung kam es hingegen - wie bei der Landesgesetzgebung - zum Verlust der Autonomie der Länder. Die Verfassung sprach folgerichtig nicht mehr von der Vollziehung der Länder, sondern nur mehr von der "Verwaltung in den Ländern“ (Überschrift des 7. Hauptstücks). Diese oblag grundsätzlich ${ }^{46}$ in den Angelegenheiten der Vollziehung des Bundes dem Landeshauptmann, in den Angelegenheiten der Vollziehung des Landes der Landesregierung und in beiden Fällen den dem Landeshauptmann unterstellten Landesbehörden. Das Amt der Landesregierung führte hinfort die Bezeichnung Landeshauptmannschaft. ${ }^{47}$ Bundes- und Landesverwaltung waren nun also stärker verzahnt, es gab, wie dies Wiederin zusammenfasst, „,keine der für einen Bundesstaat nun einmal charakteristischen Doppelgleisigkeiten mehr". ${ }^{48}$

Das Land konnte aber seine eigenen Exekutivorgane nicht mehr selbst bestellen, denn, in den Worten von Wiederin: „Wer immer etwas zu sagen hat, wird entweder wie der Landeshauptmann vom Bundespräsidenten ernannt oder muss wie der Regierungsdirektor oder die Bezirkshauptleute dem Bundeskanzler genehm sein, und zwar auf Dauer, denn auch eine Abbe-

\footnotetext{
${ }^{46}$ Soweit sie nicht durch eigene Bundesbehörden gem. Art. 120 oder nach den Gesetzen durch Selbstverwaltungskörper unter der Aufsicht des Bundes oder des Landes besorgt wurde.

${ }^{47}$ ENDER, Verfassung 18f.

${ }^{48}$ WIEDERIN, Bundesstaatlichkeit 34.
}

rufung ist jederzeit möglich“. ${ }^{49}$ Die weitreichenden Bestätigungs- und Abberufungsrechte der Zentralorgane gaben diesen damit aber auch, so Merkl, „indirekt die Möglichkeit, die landeseigene Verwaltung [...] in die Richtung der Bundespolitik zu zwingen“. ${ }^{50}$ Der Bundespräsident musste den Landeshauptmann allerdings auch dann abberufen, wenn der Landtag dies verlangte, was ein Zugeständnis an den Föderalismus darstellte, aber nur bei Vorliegen einer Zweidrittelmehrheit. Folglich konnte ein Landeshauptmann, „wenn er nur das Vertrauen der obersten Vollzugsorgane des Bundes genießt, unter allen Umständen gegen den konstanten Willen der Mehrheit der Landesrepräsentanten und umso mehr der Landesbevölkerung im Amte bleiben". ${ }^{51}$

Wien verlor als „bundesunmittelbare“ Stadt nun überhaupt den Status eines selbstständigen und gleichberechtigten Bundeslandes. Es unterstand hinfort direkt dem Bund, wenngleich einige Bestimmungen der Verfassung über die Länder auf die Stadt Wien sinngemäß anzuwenden waren (Art. 145). ${ }^{52}$

\section{Kompetenzverteilung und Bundesstaatlichkeit im Urteil der Zeitgenossen}

Die zeitgenössischen Juristen qualifizierten die Verfassung 1934 mit wenigen Ausnahmen mehr oder weniger deutlich als bundesstaatlich, nicht

\footnotetext{
${ }^{49} \mathrm{Ebd}$.

${ }^{50}$ MERKL, Verfassung 97.

${ }^{51}$ Ebd. 99.

${ }^{52}$ Auf die Stadt Wien waren sinngemäß anzuwenden die Bestimmungen der Verfassung über die rechtliche Stellung der Länder und ihrer Organe, über die Auflösung der Landtage, über das Verfahren der Gesetzgebung und Verwaltung in den Ländern, über die landesunmittelbaren Städte und über die Überprüfung der Akte der Landesorgane in Gesetzgebung und Verwaltung durch den Bundesgerichtshof.
} 
allerdings der NS-Staatsrechtler Norbert Gürke. Er kritisierte die Kompetenzregelung nicht nur als „reichlich umständlich“ und „aus den Aktenschränken der Ministerien mühsam zusammengesucht", sondern sie ginge auch "mit Vorsicht daran, alle politischen Fragen dem Bunde vorzubehalten“. Daher wäre es „unter diesen Umständen einfacher, klarer und ehrlicher, den umgekehrten Weg zu gehen und die Länderkompetenz nicht negativ, sondern positiv zu umschreiben". Es würde sich dann zeigen, „daß der ,Bundestaat" in Wahrheit kein solcher ist, sondern daß die Länder nur als Kommunalverbände höherer Ordnung übrig bleiben." Der „Bundestaat" war also Gürke zufolge durch einen „zentralistischen Verwaltungsstaat" ersetzt worden. ${ }^{53}$

Für Schuschnigg ${ }^{54}$ hingegen hatte die Verfassung 1934 unzweifelhaft „bundesstaatlichen Charakter". Der Bundesstaat setze den „staatlichen Charakter sowohl des gemeinsamen Oberstaates (Bund) als auch seiner Glieder (Länder) voraus", wobei diesen die Möglichkeit eigener Gesetzgebung und Vollziehung gewahrt sein müsse. Daher habe jede Bundesverfassung "die sogenannten Kompetenzartikel zu enthalten", wie dies in der Verfassung 1934 der Fall sei. Auch müsse den Gliedstaaten „eine Mitwirkung auf dem Gebiet der Gesetzgebung des Bundesstaates eingeräumt" sein, die „gewöhnlich durch eine Länderkammer" erfolge. Der Länderrat stelle nun - im Unterschied zu dem bisherigen, „mit einer wirklichen Länderkammer [...] nicht vergleichbar[en]“ Bundesrat - eine „echte Ländervertretung“ dar, womit gleichzeitig die „Einrichtung der früher verfassungsgesetzlich nicht vorgesehenen Länderkonferenzen [...] legalisiert" werde.

Emerich Coreth, ehemaliger VwGH-Rat, Richter am 1934 anstelle von VfGH und VwGH neu eingerichteten Bundesgerichtshof und Dienststel-

\footnotetext{
${ }^{53}$ GÜRKE, Verfassung 233ff., 254.

${ }^{54}$ SCHUSCHNIGG, Bundesverfassung 33ff.
}

lenleiter für Justiz der „Vaterländischen Front", stellte aus Anlass des Inkrafttretens der Verfassung am 1. Mai 1934 kurz und bündig fest:55 „Die Einrichtung des Bundesstaates bleibt aufrecht", denn die staatlichen Funktionen in Gesetzgebung und Vollziehung seien "auch weiterhin zwischen Bund und Ländern geteilt, allerdings mit Verstärkung der Stellung des Bundes und mit dem anerkennenswerten Bestreben, möglichst klare Grenzlinien zu ziehen“. Daher begrüßte er die Beseitigung der gespaltenen $\mathrm{Zu}$ ständigkeit (Gesetzgebung des Bundes, Vollziehung des Landes) auch ausdrücklich.

Etwas differenzierter argumentierte Ludwig Adamovich sen., ${ }^{56}$ der bald nach Inkrafttreten der Verfassung sowohl in den Staatsrat als auch Bundestag entsendet und später in den Bundesgerichtshof übernommen wurde sowie schließlich letzter Justizminister des Regimes werden sollte. Für ihn war der „Bundesstaat Österreich“ nicht unter den "hergebrachten Typus des Bundesstaates" einzureihen, sondern müsse vielmehr als „ein neuer Typus, als ein autoritär geführter Bundesstaat", gesehen werden. Der „verfassungsmäßige Aufbau“ weise zwar die „herkömmlichen Merkmale der bundesstaatlichen Organisationsform" auf, das autoritäre Prinzip wirke sich aber ,in einer dauernden Unterordnung der Organe der Länder unter den Willen der Organe des Bundes“ aus, wie dies „in diesem Maße in anderen Bundesstaaten nicht zu finden" sei.

Auch Adamovich betonte aber die „Tendenzen zur Zentralisierung" in der Kompetenzverteilung, denn es seien zwar die Kompetenzen der Länder in Gesetzgebung und Vollziehung „mit geringfügigen Aenderungen die gleichen geblieben wie bisher", und eigene Organe des Landes, der Landtag und die Landesregierung, würden auch „weiterhin in jedem Land diese Agenden

\footnotetext{
${ }^{55}$ CORETH, Grundgedanken 2.

${ }^{56}$ ADAMOVICH, Grundriss 39f.
} 
besorgen". Allerdings zeige sich nun insofern "ein grundlegender Wandel“, denn „ein Nebeneinander- oder gar ein Gegeneinanderregieren von Bund und Ländern, wie es bisher leider so häufig zu beobachten war“, würde es „in Hinkunft nicht mehr geben“, weil das oberste Landesorgan nicht nur des Vertrauens des Landtages, sondern auch des ständigen Vertrauens der obersten Bundesorgane bedürfe. Diese „Abhängigkeit des obersten Landesorganes" biete nun „,in weit größerem Maße als bisher die Gewähr dafür [...], daß ein einheitlicher Wille die ganze Verwaltung im Bund und in den Ländern beherrscht". Desgleichen sei in der Gesetzgebung der Länder "ein bedeutungsvoller Schritt auf dem gleichen Wege der Entwicklung zu verzeichnen", denn Landesgesetze, die "den Tendenzen der obersten Bundesorgane widersprechen", könne es nicht mehr geben. Damit sei „,auch für das Gebiet der Gesetzgebung die Einheitlichkeit der Entwicklung im Bund und in den Ländern gewährleistet".

Otto Ender selbst hatte Anfang April 1934 in einer Versammlung der "Vaterländischen Front" öffentlich betont, ${ }^{57}$ dass nach der neuen Verfassung "nicht alles zentralistisch eingerichtet" sei, denn ein solcher "Zentralismus würde nicht gut tun“. Er betonte auch, dass man das „Länderbewußtsein" nicht zerstören und die „Länder erhalten" wolle, wenngleich man "gar noch kein Bild“ habe, "was der ständische Aufbau für gewaltige Umwälzungen bringen“ werde, denn es könnten hinkünftig viele Kompetenzen der Landtage „in die ständische Vertretung hinübergleiten“, ja es könne „eine Zeit kommen [...], wo es keine Landtage mehr geben wird“. Man müsse - so Enders fast schon verzweifelter Appell zur Rettung der Bundesstaatlichkeit - daher darauf bestehen, „daß in den Ländern die Konzentration des ständischen Lebens stattfindet und

\footnotetext{
${ }^{57}$ Salzburger Chronik für Stadt und Land, 12. 4. 1934
} 4 (Die Länder in der neuen Verfassung).
Schwerpunkte geschaffen werden, in denen sich alles sammelt, $[\ldots]$ auf dass sie bleiben, was sie sind: Eigene, schöne, liebe Länder im großen mächtigen und herrlichen Österreich".

\section{Fazit}

Der „Bundestaat“ Österreich, wie er in der Verfassung 1934 vorgesehen war, trat in der Verfassungsrealität nicht zu Tage. Da der ständische Aufbau bis zum Ende des Regimes nicht vollendet wurde, bleibt zum einen unklar, welche Kompetenzen von Bundes- und Landesorganen an die ständischen Vertretungen übertragen worden wären. Zum anderen manifestierte sich die „verschleierte Kanzlerdiktatur“ der Verfassung, die eine Konzentration der Staatswillensbildung beim Kanzler vorsah, wobei dies aber gleichzeitig „durch ständische, bundesstaatliche und rechtsstaatliche Institutionen verdeckt" wurde, ${ }^{58}$ nicht in der Realität.

Wie die Verfassungspraxis zeigt, erfolgte die Gesetzgebung und Verwaltung freilich zumeist nicht nach den Bestimmungen der neuen Verfassung, sondern vielmehr nach der "Schattenverfassung ", 59 dem (verfassungswidrig zustande gekommenen) Ermächtigungsgesetz, ${ }^{60}$ welches die Regierung zu einer gänzlich unbeschränkten Gesetzgebung berechtigte und vom V-ÜG 1934 in Geltung belassen worden war. So ergab sich in der Praxis auch ein "eindeutiges Übergewicht" der Gesetzgebungsakte außerhalb der Verfassung, denn zwischen der Konstituierung der „Vorberatenden Organe“ und dem 11. März 1938 ergingen von 532 Bundesgesetzen 367, also fast $67 \%$, auf der Grundlage des Ermächtigungsgesetzes. ${ }^{61}$ Zudem hatte die Bundesregierung nach der "Übergangsverfassung“ in Form

\footnotetext{
58 WeLAN, NeISSER, Bundeskanzler 48.

${ }^{59}$ WIEDERIN, Bundesstaatlichkeit 32.

${ }^{60}$ BGBl. I 255/1934.

${ }^{61}$ WOHNOUT, Regierungsdiktatur 305f.
} 
des V-ÜG auch den Zugriff auf sämtliche Landesverwaltungsorgane, was die Bundesstaatlichkeit auf die Ebene einer hohlen Phrase reduzierte. ${ }^{62}$ Aber auch das zentrale Element der Berufsstände blieb bis zum Ende des Regimes ein bloßes „Potemkisches Dorf“ ${ }^{\prime 63}$

So konnte in der Praxis die Kanzlerdiktatur also, ohne Berücksichtigung jedweder Kompetenzverteilung, völlig unverschleiert praktiziert werden - bedeutet doch "Diktatur", so der damals in Berlin lehrende österreichische Staatsrechtler Hermann Heller, ${ }^{64}$ „immer zentralistische Gewaltvereinigung in der Hand des Diktators", also das Gegenteil sowohl des in der Verfassung verankerten Korporativismus als auch der in Grundzügen noch vorgesehenen Bundesstaatlichkeit.

\section{Korrespondenz:}

Prof. Dr. Ilse ReITER-ZATLOUKAL

Universität Wien

Institut für Rechts- und Verfassungsgeschichte

Schottenbastei 10-16 (Juridicum)

1010 Wien

ilse.reiter-zatloukal@univie.ac.at

ORCID-Nr. 0000-0002-6420-4083

62 Siehe für die zentralen Merkmale des Bundesstaates nach Ansicht des VfGH den Beitrag von Anna Gamper in diesem Band.

${ }^{63}$ ReIter-ZatloukaL, ",Selbstverwaltung“.

${ }^{64}$ HELLER, Rechtsstaat 22

\section{Abkürzungen:}

MRP Ministerratsprotokolle

V-ÜG Verfassungs-Überleitungsgesetz

Siehe auch das allgemeine Abkürzungsverzeichnis:

[http://www.rechtsgeschichte.at/media/abk.pdf]

\section{Literatur und Quellen:}

Ludwig ADAMOVICH, Grundriss des österreichischen Staatsrechtes (Verfassungs- und Verwaltungsrechtes, Wien $\left.{ }^{3} 1935\right)$.

Emerich CORETH, Grundgedanken der ständischen Verfassung, Wiener Zeitung, 1. 5. 1934, Nr. 2, 2.

Otto ENDER, Die neue Österreichische Verfassung mit dem Text des Konkordates (= Der neue Staat 1, Wien-Leipzig 1934).

Michael EtLINGER, Die Landesverfassungen 1934 (rechtswiss. Diss. Univ. Wien 2001).

Norbert GÜRKE, Die österreichische „Verfassung 1934", in: Archiv des öffentlichen Rechts NF 25 (1934) 178-255

Hermann HeLLER, Rechtsstaat oder Diktatur (Geschichte und Staat in Geschichte und Gegenwart 68, Tübingen 1930).

Hugo JÄCKL, Die Verfassung 1934, in: Österreichisches Verwaltungsblatt, Beilage zur Wiener Zeitung vom 16. Mai 1934, 105-112.

Max Kulisch, Kritische Studien zu einigen Kompetenzartikeln der österreichischen Bundesverfassung von 1934. Ein Beitrag zur Auslegung der Verfassung, in: Österreichisches Verwaltungsblatt (1938) 168-175.

Adolf MERKL, Das neue Verfassungsrecht, in: Juristische Blätter 63 (1934) 201-206, 265-270, 290-299, 357-359.

DERS., Die ständisch-autoritäre Verfassung Österreichs. Ein kritisch-systematischer Grundriß (Wien 1935).

Rudolf NeCK, Adam WandruszKa (Hgg.), Protokolle des Ministerrates der Ersten Republik 1918-1938, VIII/5 (Wien 1984), VIII/6 (Wien 1985).

Martin F. POLASCHEK, Der Föderalismus in der Verfassung 1934, in: Geschichte und Gegenwart 12/3 (1993) 141-178.

Ilse ReITER-ZATLOUKAL, Parlamentarismus im Fadenkreuz. Demokratiekonzepte und (Anti-)parlamentarismus in Österreich 1918-1933/34, in: Parlamentsdirektion (Hg.), Staats- und Verfassungskrise 1933 (Wien-Köln-Weimar 2014) 19-50. 
DIES., Die Rechtsstellung der Länder 1933 bis 1938. Verfassungsrechtliche Rahmenbedingungen und Rechtsrealität, in: Martin SCHENNACH (Hg.), Rechtshistorische Aspekte des österreichischen Föderalismus. Beiträge zur Tagung an der Universität Innsbruck am 28. und 29. November 2013 (Wien 2015) 147-182.

DIES., Berufsständische „Selbstverwaltung“ in der österreichischen Diktatur 1933-1938. (Regulierte) Selbstregulierung als Potemkinsches Dorf, in: Peter Collin u.a. (Hgg.), Regulierte Selbstregulierung in der westlichen Welt im späten 19. und frühen 20. Jahrhundert (= Studien zur europäischen Rechtsgeschichte. Veröffentlichungen des MaxPlanck-Instituts für europäische Rechtsgeschichte 290, Frankfurt a.M. 2014) 213-234.

DIES., Staatsbürgerschaftsrecht in Österreich 19331938, in: BRGÖ 2 (2011) 291-316.

Kurt Schuschnigg (Hg.), Die neue Bundesverfassung für Österreich samt Übergangsbestimmungen (= Tagblatt-Bibliothek 838/840, Leipzig-Wien-Berlin 1936).
DERS., Die Verfassung des Bundesstaates Österreich (Leipzig-Wien-Berlin 1934).

Josef WERNDL, Die Kompetenzverteilung zwischen Bund und Ländern. Ihre Ausgangslage, Entwicklung und Bedeutungsverschiebung auf der Grundlage des Bundes-Verfassungsgesetzes von 1920 (= Schriftenreihe des Instituts für Föderalismusforschung 32, Wien 1984).

Manfried WELAN, Heinrich NEISSER, Der Bundeskanzler im österreichischen Verfassungsgefüge (Wien 1971).

Ewald WIEDERIN, Christliche Bundesstaatlichkeit auf ständischer Grundlage: Eine Strukturanalyse der Verfassung 1934, in: Ilse ReITER-ZATlOUKAL, Christiane ROTHLÄNDER, Pia SCHÖLNBERGER (Hgg.), Österreich 1933-1938. Interdisziplinäre Annäherungen an das Dollfuß-/Schuschnigg-Regime (WienKöln-Weimar 2012) 31-41.

Helmut WoHNOUT, Regierungsdiktatur oder Ständeparlament? Gesetzgebung im autoritären Österreich (Wien 1993). 